\title{
Pseudomonas aeruginosa infection in respiratory samples in children with neurodisability - to treat or not to treat?
}

\author{
Elizabeth Gregson ${ }^{1} \cdot$ Lowri Thomas $^{1} \cdot$ Heather E Elphick ${ }^{1}$ (I) \\ Received: 28 October 2020 / Revised: 5 March 2021 / Accepted: 10 March 2021 / Published online: 6 April 2021 \\ (C) The Author(s) 2021
}

\begin{abstract}
The objective was to investigate the prevalence of Pseudomonas aeruginosa (PA) in patients with complex neurodisability and current treatment practice in our centre in order to inform future guidelines. A retrospective case note review was undertaken at a tertiary children's hospital. One hundred sixty-two patients (mean age 11.7 years) with a primary diagnosis of neuromuscular disease (NMD) or severe cerebral palsy (CP) and a respiratory sample sent for analysis during the study period were studied. Associations between PA in respiratory samples and diagnosis, long-term ventilation, presence of a gastrostomy or a tracheostomy, antibiotic choice, clinical deterioration and adverse events were analysed. Twenty-five (15\%) had one or more PA isolate in respiratory samples. There was a significant association between PA in respiratory samples and tracheostomy ( $p<0.05)$. In $52 \%$ samples, multiple pathogens co-existed. There was no significant association between choice of antibiotic and clinical outcome but when antibiotics were changed to specific PA antibiotics during the course of the illness, all resulted in clinical improvement. Twenty-six episodes involving 8 patients with recurrent admissions involved PA organisms that were resistant to one or more antibiotics.

Conclusions: A larger prospective study may establish clearer criteria for guideline development. Techniques such as point-of-care testing to identify virulent strains of PA may improve patient outcomes and prevent the development of antibiotic resistance in the future.

\section{What is Known:}

- Children with complex neurodisability are at increased risk of respiratory morbidity and of infection with gram-negative organisms such as Pseudomonas aeruginosa.

- There are currently no guidelines to inform treatment choices in this group of vulnerable children.

What is New:

-15\% children in this study population had Pseudomonas aeruginosa in respiratory samples during a 12-month period, the majority of whom did not require critical care treatment. Thirteen of these children had a tracheostomy in situ and 12 did not.

-In those that deteriorated clinically or developed antibiotic resistant organisms, earlier detection and targeted treatment of Pseudomonas aeruginosa may have prevented deterioration.
\end{abstract}

\section{Communicated by Peter de Winter}

Heather E Elphick

h.elphick@nhs.net

Elizabeth Gregson

elizabeth.gregson@nhs.net

Lowri Thomas

lowrithomas@nhs.net

1 Department of Respiratory Medicine, Sheffield Children's NHS Foundation Trust, Sheffield, UK
Keywords Pseudomonas aeruginosa · Child · Neurodisability · Antibiotic resistance $\cdot$ Point-of-care testing

\section{Introduction}

Children with complex neurodisability such as neuromuscular disorders (NMD) and cerebral palsy (CP) are at increased risk of respiratory morbidity due to factors such as gastrooesophageal reflux, kyphoscoliosis, muscle weakness, secretions and poor cough $[1,2] .25 \%$ patients with cerebral palsy of severity 4-5 on the GMFCS (Gross Motor Function Classification System) have chronic respiratory problems [3] 
including cough and wheeze, obstructive sleep apnoea, cough on drinking and respiratory signs on examination [4]. Pneumonia, often due to aspiration, is a common cause of hospital admission, intensive care admission and death in these patients [5-8]. The more severely affected children are admitted 7 times more often, with 9.5 times as many admitted days as the normal population [9] Data suggest that pneumonia is responsible for 39\% deaths in children with CP [10].

Pseudomonas aeruginosa (PA) is a gram-negative bacillus and is well known to cause infections and lead to colonisation of the airways. In Cystic Fibrosis, PA has been shown to bind to the respiratory epithelium [11-14]. It has been postulated from this evidence that PA also binds to the respiratory epithelium eroded by chronic subclinical oral aspiration and/or reflux causing chronic PA lower airway infection in patients with neurodisability [15]. There is a higher incidence of PA in children with $\mathrm{CP}$ in paediatric intensive care units (PICU) [10]; children with CP who are infected with PA are significantly more likely to have severe illness, be admitted to PICU and are also more likely to have prolonged or recurrent hospital admissions [15]. A review of children in PICU in Liverpool, UK reported that $89 \%$ children with CP carried PA or Klebsiella, compared with $55 \%$ without $\mathrm{CP}$ and $47 \%$ carried antibiotic resistant bacteria [10]. Treatment of PA in PICU is often problematic and the emergence of resistant organisms can occur as early as 8 days following admission [16].

Treatment for acute PA infection requires either a specific oral antibiotic, such as ciprofloxacin, which can exacerbate seizures or alter feeding regimes, or intravenous antibiotic treatment, which requires cannulation and hospitalisation. Both options lead to potential additional morbidity for the patient, including antimicrobial resistance [17]. Decision to treat with intravenous antibiotic treatment at an early stage in the illness may later prove unnecessary if the infection was in fact virus-driven. Conversely, delayed treatment for PA infection can result in severe clinical deterioration and escalation of treatment requirements. There are no universally agreed guidelines on when to treat PA, treatment choices are often empirical [18] or extrapolated from other conditions with a more established evidence base, for example cystic fibrosis [19]. There is a wide range of practice in terms of treating PA, with some clinicians treating only if symptomatic, some not treating at all and others treating regardless of symptoms [20].

The aim of this study was to investigate the prevalence of PA in patients with complex neurodisability and current treatment practice in our centre in order to inform future guidelines. Associations between clinical factors and treatment choices were analysed to establish whether these influenced clinical outcomes during an acute respiratory exacerbation.

\section{Methods}

\section{Study population}

The study population was all patients in the local region of our NHS Trust who:

1. Had a diagnosis of NMD or CP GMFCS 4 or 5

2. Did not have a primary diagnosis of cystic fibrosis or chronic lung disease

3. Had a microbiology culture on a respiratory sample during the study period

A comprehensive list of children with neuromuscular disease and cerebral palsy GMFCS 4 or 5 under the care of our NHS Trust was obtained from three databases kept up to date by the physiotherapy team working with children with neurodisability. A further database of children using overnight long-term ventilation (LTV) was obtained from the nursing team working with this patient group.

A list of patients that had a positive growth of PA on sputum or airway secretions between $01 / 01 / 17$ and $31 / 12 / 17$ was obtained from the microbiology lab records and amalgamated with the patient databases to find patients that fulfilled the study criteria.

\section{Data collection}

The study was undertaken during the period 01/01/17-31/12/ 17 at a UK tertiary children's hospital. All patients listed in the database who had also had a microbiology sample positive for PA during the study period were reviewed to elicit treatment decisions and clinical outcomes.

Data collection was as follows:

- Demographic information - age, gender, primary diagnosis, presence of tracheostomy, gastrostomy, use of LTV;

- PA infection-source of isolation; evidence of preexisting infection/colonisation such as long-term antibiotics; other pathogens isolated during the illness being investigated;

- Treatments used, including type of antibiotic, timing relative to isolation of PA and antibiotic changes;

- Clinical information relating to a clinical deterioration or improvement, change in clinical condition after antibiotic changes;

- Adverse events as a result of treatment - side effects to antibiotics, organism resistance.

Some patients had several samples taken within the 12month study period. A "PA positive clinical episode" was therefore defined as one or more positive isolates of PA within 1 week, as a surrogate measure of a single acute clinical 


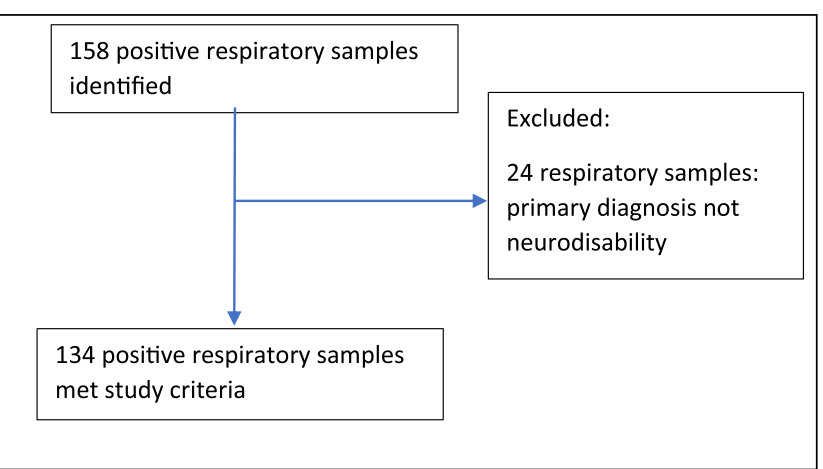

Fig. 1 Flowchart of the positive samples identified in the microbiology lab records during the 12-month study period

illness. Samples that were positive more than 1 week apart were assumed to be a separate illness and/or a colonisation.

"Clinical deterioration" was defined as a persistent increase (for more than $12 \mathrm{~h}$ ) of oxygen requirements, ventilatory support, any inotropic support/fluid resuscitation. If the PA was not treated and a clinical deterioration occurred within a week of this decision being made, this was counted as a deterioration due to no treatment being given. The term "treatment" is used specifically to only mean antibiotic treatment. Additional treatment for example supportive measures or respiratory support was not looked at specifically but only to establish if there was clinical deterioration. Treatment categories were antibiotics that are used specifically to treat PA such as ciprofloxacin or tazocin or non-specific antibiotics such as amoxicillin or azithromycin that may be used for a lower respiratory tract infection but not specifically to cover PA. This definition was chosen to apply standardisation within the cohort of patients analysed, given the retrospective design of the study.

\section{Data analysis}

The anonymised data collection was stored in an Excel spreadsheet. Chi-square analysis was used to test whether diagnostic and clinical variables were statistically independent of one another (Excel). Analyses for type of antibiotic and clinical outcomes were repeated for patients without a tracheostomy. Statistical significance was considered to be at the level of $p<0.05$.

\section{Results}

Two hundred twenty-six patients were identified from the clinical databases and 158 positive samples for PA were identified in the microbiology lab records (Fig. 1). Patients with cystic fibrosis were excluded prior to searching the database; however, a further 24 patients were excluded as the primary underlying diagnosis was not a neurodisability. Therefore, 134 positive samples fulfilled the study criteria, with isolates grown from tracheostomy
Fig. 2 Neuromuscular diagnoses in patients infected with PA in respiratory samples $(n=19)$

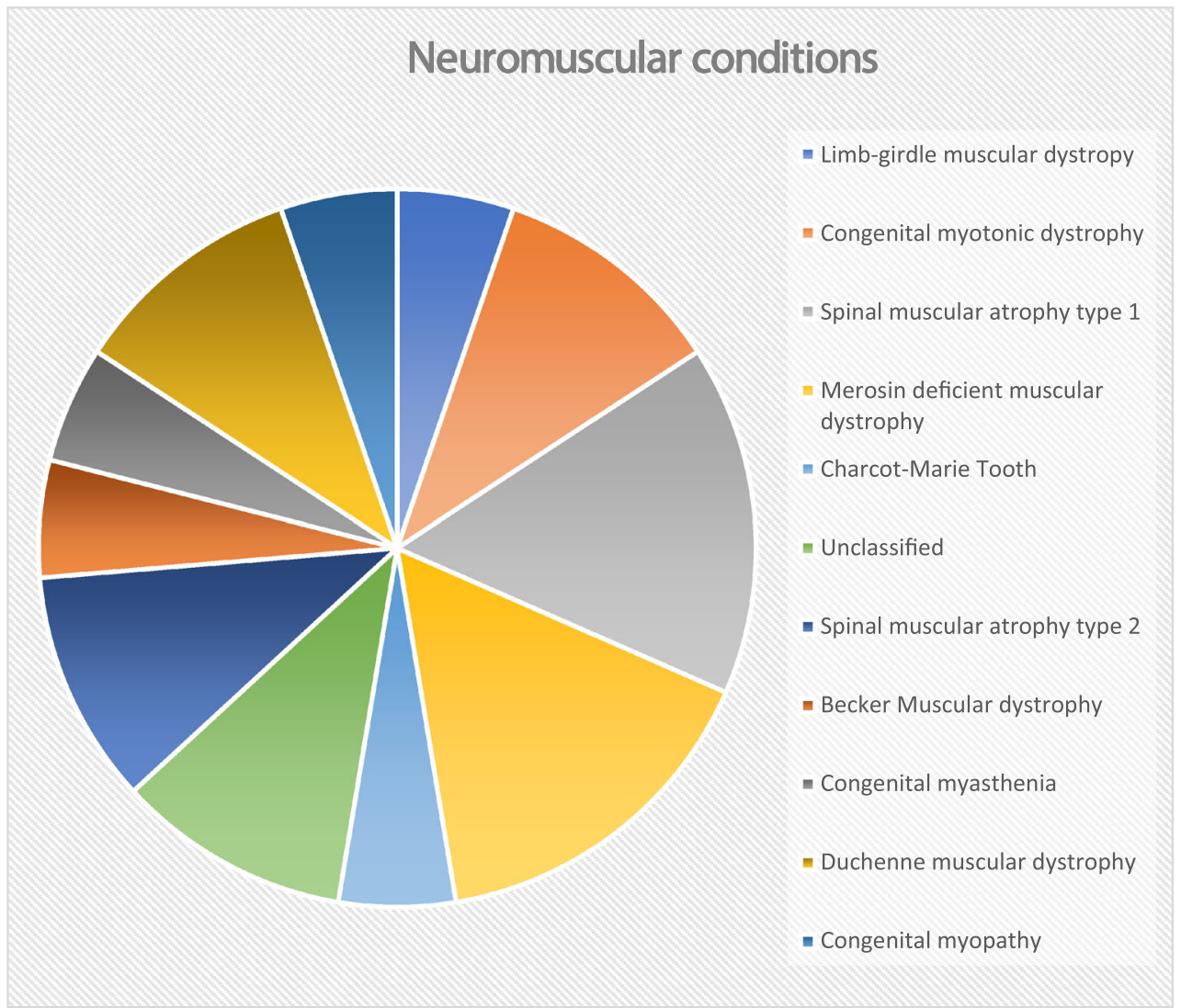


Table 1 Analysis of patients in whom a respiratory sample was analysed during the study period $(n=162) . N M D$, neuromuscular disease; $C P$, cerebral palsy; GMFCS, Gross Motor Function Classification System; $P A$, pseudomonas aeruginosa; $N I V$, non-invasive ventilation

\begin{tabular}{llll}
\hline Patient groups & PA positive & PA negative & $p$ value (chi-square) \\
\hline Total patients $(n=162)$ & $25 / 162(15 \%)$ & $137 / 162(85 \%)$ & \\
Mean age (years) & 13.7 & 11.5 & \\
Gender (male:female) & $18: 7$ & $81: 56$ & \\
Subgroups by disease group: & & & \\
Total NMD $(n=128)$ & $19 / 128(15 \%)$ & $109 / 128(85 \%)$ & \\
NMD with tracheostomy $(n=25)$ & $10 / 25(40 \%)$ & $15 / 25(60 \%)$ & \\
NMD without tracheostomy $(n=103)$ & $9 / 103(9 \%)$ & $94 / 103(91 \%)$ & \\
NMD with NIV $(n=18)$ & $1 / 18(6 \%)$ & $17 / 18(94 \%)$ & \\
NMD without NIV $(n=110)$ & $18 / 110(16 \%)$ & $92 / 110(84 \%)$ & \\
NMD with gastrostomy $(n=43)$ & $2 / 43(5 \%)$ & $41(95 \%)$ & \\
NMD without gastrostomy $(n=85)$ & $17 / 85(20 \%)$ & $68 / 85(80 \%)$ & \\
Total CP GMFCS 4-5 $(n=34)$ & $6 / 34(18 \%)$ & $28 / 34(82 \%)$ & \\
CP GMFCS 4-5 with tracheostomy $(n=8)$ & $3 / 8(38 \%)$ & $5 / 8(62 \%)$ & \\
CP GMFCS 4-5 no tracheostomy $(n=26)$ & $3 / 26(12 \%)$ & $23 / 26(88 \%)$ & \\
CP with NIV $(n=9)$ & $2 / 9(22 \%)$ & $7 / 9(78 \%)$ & \\
CP without NIV $(n=25)$ & $4 / 25(16 \%)$ & $21 / 25(84 \%)$ & \\
CP with gastrostomy $(n=23)$ & $4 / 23(17 \%)$ & $19 / 23(83 \%)$ & \\
CP without gastrostomy $(n=11)$ & $2 / 11(18 \%)$ & $9 / 11(82 \%)$ & \\
Subgroups by chronic therapy: & & & \\
NIV $(n=27)$ & $3(11 \%)$ & $24(89 \%)$ & \\
Tracheostomy $(n=33)$ & $13(39 \%)$ & $20(61 \%)$ & \\
Gastrostomy $(n=66)$ & $6(0.09 \%)$ & $60(90.944 \%)$ & \\
\hline
\end{tabular}

${ }^{1}$ Chi-square is testing the number of subjects with NMD/number of subjects with CP with PA vs. number of subjects with NMD/number of subjects with $\mathrm{CP}$ who did not have PA

${ }^{2}$ Chi-square is testing the number of subjects with tracheostomy/number of subjects without tracheostomy with PA vs. number of subjects with tracheostomy/number of subjects without tracheostomy who did not have PA

${ }^{3}$ Chi-square is testing the number of subjects using NIV/number of subjects not using NIV with PA vs. number of subjects using NIV/number of subjects not using NIV who did not have PA

${ }^{4} \mathrm{Chi}$-square is testing the number of subjects with gastrostomy/number of subjects without gastrostomy with PA vs. number of subjects with gastrostomy/number of subjects without gastrostomy who did not have PA secretions, endotracheal tube secretions, nasopharyngeal aspirate or secretions, cough swab or sputum.

Of the 181 patients with neuromuscular disease, respiratory samples were sent for bacterial analysis from 128 patients (71\%). Nineteen of these patients had positive samples for PA $(15 \%)$, of whom $11(58 \%)$ were positive on more than one isolate within the 12-month period. Underlying conditions in the 19 patients with positive samples are shown in Fig. 2 and were muscular dystrophies: limb girdle muscular dystrophy $(n=1)$, merosin-deficient muscular dystrophy $(n=3)$, Duchenne muscular dystrophy $(n=2)$, Becker muscular dystrophy $(n=1)$; Spinal muscular atrophy type $1(n=3)$, Spinal muscular atrophy type $2(n=2)$, unclassified $(n=2)$, congenital myasthenia $(n=1)$, congenital myotonic dystrophy $(n=2)$, Charcot-Marie Tooth $(n=1)$ and congenital myopathy $(n=1)$.

A total of 113 patients with cerebral palsy were identified. Of these, 45 had a GMFCS score of 4-5 and of these, respiratory samples were sent for bacterial analysis during the study period from 34 (76\%). Six patients had positive samples for PA $(18 \%)$, of whom two had more than one positive isolate within the 12-month period.

Table 1 shows the analysis of patients in whom a respiratory sample was taken during the study period $(n=162)$.
Table 2 Clinical outcomes based on decision to treat $(n=60)$. There was no significant association between type of antibiotic and clinical outcome. $p=0.06$ (chisquare)

\begin{tabular}{llll}
\hline Outcome & No antibiotics & Non-specific antibiotics & PS specific antibiotics \\
\hline Did not deteriorate & $25(89 \%)$ & $10(59 \%)$ & $11(73 \%)$ \\
Deteriorated & $3(11 \%)$ & $7(41 \%)$ & $4(27 \%)$ \\
Total & 28 & 17 & 15 \\
\hline
\end{tabular}


Table 3 Clinical outcomes based on decision to treat $(n=20)$ in only those patients without a tracheostomy. There was no significant association between type of antibiotic and clinical outcome. $p=0.36$ (chi-square)

\begin{tabular}{llll}
\hline Outcome & No antibiotics & Non-specific antibiotics & PS specific antibiotics \\
\hline Did not deteriorate & $2(40 \%)$ & $7(64 \%)$ & $1(25 \%)$ \\
Deteriorated & $3(60 \%)$ & $4(36 \%)$ & $3(75 \%)$ \\
Total & 5 & 11 & 4 \\
\hline
\end{tabular}

The age range for the full cohort was 2 months to 17 years (mean 11.7 years) with 99 males and 63 females. Twentyseven were using non-invasive ventilation (NIV) via a facemask, 10 were ventilated via tracheostomy, 23 had a tracheostomy with no ventilation and 66 had a gastrostomy tube for feeding. Of the 25 patients that had a PA isolate, $19(76 \%)$ had NMD and $6(24 \%$ had CP. Thirteen $(52 \%)$ had a tracheostomy (of whom 10 were receiving overnight ventilation), 3 (12\%) were using NIV and $6(24 \%)$ had a gastrostomy.

There was no significant association between diagnosis of NMD or CP and PA analysis, There was no significant association between use of NIV or gastrostomy and PA analysis but there was a significant association between presence of a tracheostomy and PA positive samples $(p<0.05)$.

After exclusion of duplicate samples (from the same patient during the same clinical episode), 62 PA positive clinical episodes were included from the 25 individual patients. Table 2 shows the clinical outcomes of the episodes treated with antibiotics specific to PA, those treated empirically with non-specific antibiotics and those that received no antibiotics for the 60 episodes for which an outcome could be identified. There was no significant association between type of antibiotic and clinical outcome $(p=0.06)$.

There were 20 clinical episodes in 13 individual patients who did not have a tracheostomy. Table 3 shows their clinical outcomes. The children that had a PA isolate without a tracheostomy represented 9/19 of those with NMD (16 clinical episodes) and 3/6 of those with CP (4 clinical episodes).

Table 4 illustrates the ten episodes in which antibiotics were started or changed after clinical deterioration to one that was specific and sensitive for the PA isolated during the admission after receipt of microbiology results. Thereafter all patients improved.

In $52 \%$ samples, multiple pathogens co-existed (Fig. 3).

Tables 5, 6 and 7 show the results of the association between isolated PA infections and multiple pathogens, new PA infection and prior colonisation and the use of prophylactic nebulised colomycin.

Of the 62 episodes, 28 samples grew PA alone and 34 grew multiple pathogens. In 58 episodes, PA had been grown on previous respiratory samples and of those, nebulised colomycin was being used in 13 episodes. There was no significant association between clinical deterioration and the presence of PA alone or multiple respiratory pathogens. There was a significant association between the presence of PA in previous samples and clinical deterioration with deterioration being more likely to occur in those that had previously grown PA. There was no significant association between clinical deterioration and the use of colomycin nebulisers, although most (12/13) patient taking colomycin did not deteriorate.

In the 15 episodes in which there was a clinical deterioration, there was no association between antibiotic choice and presence of single or multiple pathogens, previous PA growth or use of colomycin nebulisers.

No adverse clinical effects were documented as a direct result of the antibiotics. Of the 62 PA positive clinical episodes, 26 episodes were associated with PA organisms that were documented as being resistant to one of more antibiotics. These episodes were from 8 individual patients, all of whom had recurrent admissions. Table 8 shows the bacteriological and clinical outcomes of these patients.

\section{Discussion}

This study has shown that of 226 patients with MND or severe CP under the care of a tertiary Children's Trust, 162 had a respiratory sample sent during a 12-month period and 25 $(15 \%)$ of these had one or more PA isolates. There was a significant association with presence of a tracheostomy $(p<0.05)$. In $52 \%$ samples multiple pathogens co-existed. Whilst in 10 clinical episodes, there was an improvement after commencing PA specific antibiotics; of the 60 PA positive clinical episodes, there was no significant association between choice of antibiotic and clinical outcome.

Table 4 Clinical outcomes in PA positive clinical episodes in which patients deteriorated and had antibiotics changed to PA specific antibiotics based on change in treatment after microbiology result $(n=10)$

\begin{tabular}{lll}
\hline Outcome & $\begin{array}{l}\text { Initial treatment: } \\
\text { No antibiotics }\end{array}$ & $\begin{array}{l}\text { Initial treatment: } \\
\text { Non-specific antibiotics }\end{array}$ \\
\hline Improved after change & 2 & 8 \\
Deteriorated further & 0 & 0 \\
Total & 2 & 8 \\
\hline
\end{tabular}




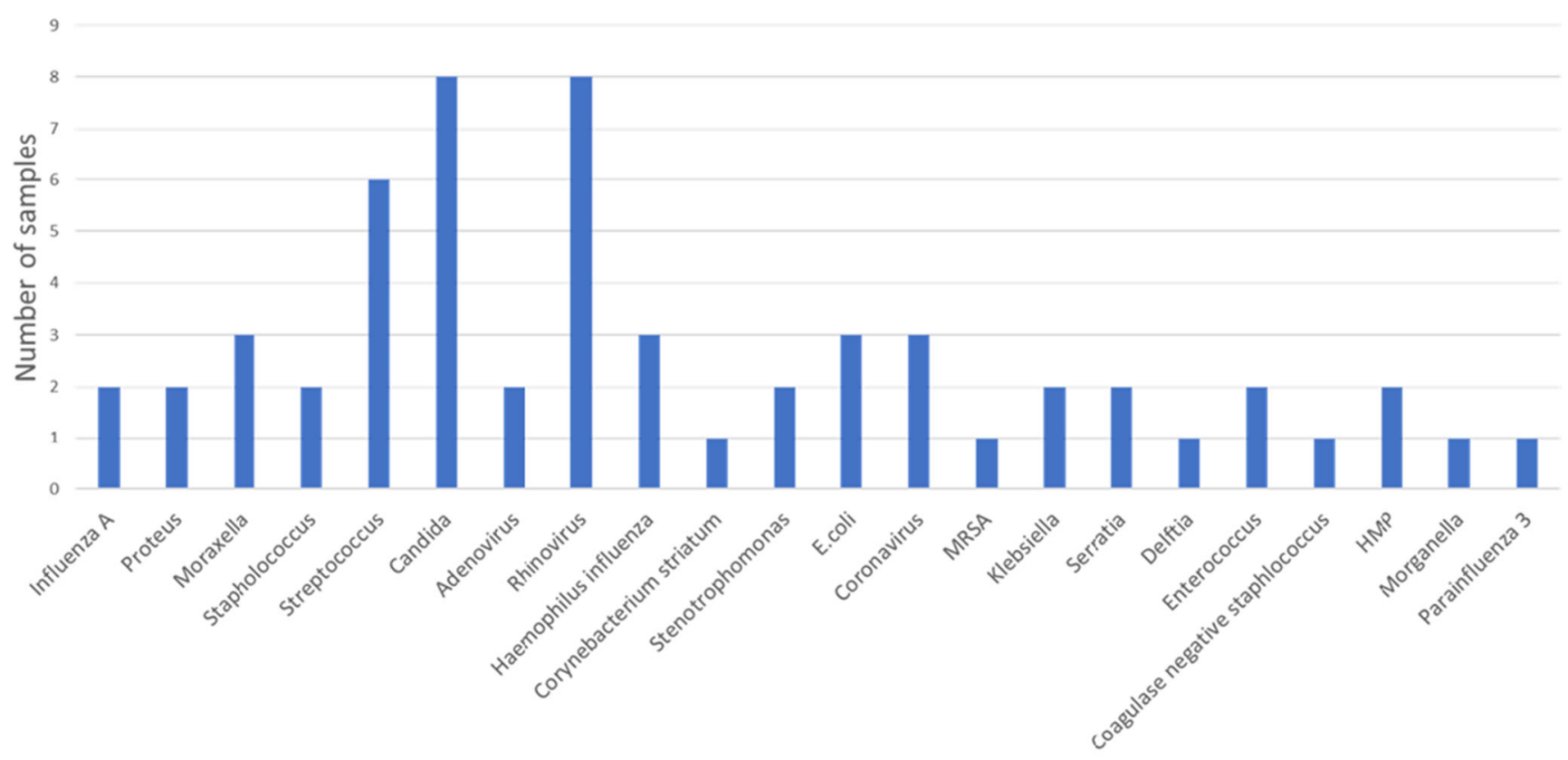

Type of pathogen

Fig. 3 Additional pathogens grown in addition to PA

The finding that tracheostomy samples are associated with PA isolates is consistent with other studies that show a predominance of Gram-negative organisms in this patient population [21-25]. Biofilms are formed when the bacteria adhere strongly to the surfaces of the tubes, providing protection to the bacteria against antibiotic treatment. The relationship between presence of PA in the airway and respiratory health or the need for antibiotic treatment is not fully understood. Data from Russel et al. suggest that the early colonisation with $P$. aeruginosa after tracheostomy is associated with higher morbidity [26]; however, McCaleb et al. [27] suggest a poor correlation, reflected by current practice in which many patients are only treated with antibiotics when they are symptomatic and there is clear clinical evidence of bacterial infection.

The majority of patients did not have a significant clinical deterioration regardless of treatment choice and the largest group had no treatment. This may reflect the possibility that some of these samples could have been taken for surveillance purposes, although this is not routine practice in our centre [28]. Alternatively, if patients were

Table 5 Association between deterioration and presence of PA alone or multiple respiratory pathogens $(p=0.64)$

\begin{tabular}{llll}
\hline & PA alone & Multiple pathogens & Total \\
\hline Clinical deterioration & 6 & 9 & 15 \\
No clinical deterioration & 22 & 25 & 47 \\
Total & 28 & 34 & 62 \\
\hline
\end{tabular}

only mildly unwell when samples were taken, it is likely that the clinician would have a watch and wait approach, to avoid unnecessary morbidity associated with PA treatment. Whilst information on additional cultures such as urine was not collected, a large number of co-existing pathogens were identified and there was no significant association between clinical deterioration and multiple respiratory pathogens. It is difficult to draw conclusions from this, however, as the microbiological cause of those that did deteriorate remains unclear. It is possible that for many, the relatively mild nature of their illness was predominantly the natural history of a viral infection, indicating that the PA was not the pathogen driving the illness, and merely reflected colonisation. There was a significant association between the presence of PA in previous samples and clinical deterioration with deterioration being more likely to occur in those that had previously grown PA. This perhaps indicates that deterioration in this chronically ill patient group was more likely associated with the pre-existing poor condition of the child.

Table 6 Association between deterioration and presence of PA in previous respiratory samples $(p=0.0002)$

\begin{tabular}{llll}
\hline & Previous PA & New PA & Total \\
\hline Clinical deterioration & 11 & 4 & 15 \\
Clinical deterioration & 47 & 0 & 47 \\
Total & 58 & 4 & 62 \\
\hline
\end{tabular}


Table 7 Association between deterioration and prior use of nebulised colomycin $(p=0.19)$

\begin{tabular}{llll}
\hline & Colomycin & No colomycin & Total \\
\hline Clinical deterioration & 1 & 14 & 15 \\
Clinical deterioration & 12 & 35 & 47 \\
Total & 13 & 49 & 62 \\
\hline
\end{tabular}

The largest group to deteriorate was that in whom nonspecific antibiotics were initially given, but numbers were small and therefore little conclusion can be drawn from these figures. Due to the small sample size $p$ values were not adjusted for multiple comparisons. The improvement seen in the ten patients that had PA-specific antibiotics started following microbiology results may indicate that the ability to isolate a specific pathogen earlier in the illness may prevent further deterioration. It was not possible to ascertain from this study whether the use of PA specific antibiotics early in the illness prior to receipt of microbiology results was detrimental, but no adverse effects were documented as a direct result of the antibiotics. Antibiotic resistant PA organisms were isolated from eight patients, with those developing resistance or remaining multiresistant being more likely to require escalation to intensive care treatment. These findings cannot be attributed to the antibiotic alone, as improvement with time and other treatment changes will have contributed to the outcome.

The retrospective nature of our case note analysis was a limitation of this study which precludes drawing conclusions as to the timing of treatment aimed at PA and prior or new colonisation (as surveillance cultures are not routine practice). More definitive analysis would be enabled by a prospective study to look at outcomes in more detail as well as other possible confounders including the severity of neurodisability and co-morbid conditions. In the absence of clinical guidelines many of the results were reliant on individual clinician judgements in terms of IV versus oral antibiotics and whether broad-spectrum or PA specific antibiotics were commenced, creating a potential for bias. In the patients that did not have a respiratory sample sent for analysis, it was assumed that this was due to good respiratory health and absence of clinical indicators to raise concerns for infection. As none of these clinically well patients were actively sampled, the results of this study may underestimate the true prevalence.

In conclusion, in a 12-month retrospective study of patients with NMD or CP, the majority of the $15 \%$ patients with respiratory PA isolates did not significantly deteriorate clinically and outcomes in relation to antibiotic treatment choices were unclear due to small patient numbers. A large number of co-existing pathogens was identified and it is possible that for many the relatively mild nature of their illness was predominantly the natural history of a viral infection, indicating that the PA was not the pathogen driving the illness. The ability to identify the presence of PA early in the illness may have resulted in earlier clinical improvement in the patients that were initially treated with non-specific antibiotics. Antibiotic resistance in patients with recurrent admissions could be reduced by introducing targeted antibiotic treatment, although this study was not able to show this. A larger prospective study may establish clearer criteria for guideline development and more sophisticated methods to direct treatment choices are needed. Techniques such as the ability to identify virulent strains of PA using point-ofcare testing may improve patient outcomes and prevent the development of antibiotic resistance in the future.

Table 8 Bacteriological and clinical outcomes of patients with antibiotic resistant PA. $H D U$, high dependency unit; $P I C U$, paediatric intensive care unit. Antibiotic resistance: resistance pattern during

treatment of PA episode described. Bacteriological outcome: resistance patterns of subsequent growths of PA after treatment

\begin{tabular}{|c|c|c|c|c|}
\hline & Antibiotic Resistance & Treatment Changes & Bacteriological Outcome & Clinical outcome \\
\hline Patient 1 & Ceftazidime & Changed to tazocin & Resistant to gentamicin & Improvement \\
\hline Patient 2 & Fully sensitive & Treated with cipro & Resistant to gentamicin & Escalated to HDU \\
\hline Patient 3 & Multiresistant & $\begin{array}{l}\text { Treated with series of antibiotics, } \\
\text { including: tazocin, tobramycin, } \\
\text { meropenem }\end{array}$ & Remained multiresistant & $\begin{array}{l}\text { Multiple PICU } \\
\text { admissions }\end{array}$ \\
\hline Patient 4 & Meropenem & Tazocin & Multiresistant & Escalated to PICU \\
\hline Patient 5 & Fully sensitive & Cephalexin & Resistant to cipro & Stayed at home \\
\hline Patient 6 & Multiresistant & Meropenem, tazocin & Multiresistant & Escalated to PICU \\
\hline Patient 7 & Fully sensitive & $\begin{array}{l}\text { Treated with series of antibiotics, } \\
\text { including: tazocin, ciprofloxacin, } \\
\text { meropenem }\end{array}$ & Multiresistant & Escalated to PICU \\
\hline Patient 8 & Fully sensitive & None & Multiresistant & Stayed at home \\
\hline
\end{tabular}


Abbreviations CP, Cerebral palsy; GMFCS, Gross Motor Function Classification System; HDU, High dependency unit; IV, Intravenous; LTV, Long-term ventilation; NIV, Non-invasive ventilation; NMD, Neuromuscular disease; PA, Pseudomonas aeruginosa; PICU, Paediatric intensive care unit

Author's contributions Elizabeth Gregson contributed to the design of the work; the acquisition, analysis and interpretation of data for the work; drafted paper and approved the final version to be published. Lowri Thomas contributed to the acquisition of data for the work, revised the paper critically and approved the final version to be published. Heather E Elphick contributed to the conception and design of the work, analysis and interpretation of data, critical revision of the paper and approved the final version to be published. All authors agree to be accountable for all aspects of the work in ensuring that questions related to the accuracy or integrity of any part of the work are appropriately investigated and resolved.

Funding This study was supported by The Children's Hospital Charity, Sheffield Children's NHS Foundation Trust.

Availability of data and material Available on request

Code availability Not applicable

\section{Declarations}

Ethics approval Service evaluation approvals were obtained (registration number SE1272).

\section{Consent to participate Not applicable}

Consent for publication All authors consent.

Conflict of interest The authors declare no competing interests.

Open Access This article is licensed under a Creative Commons Attribution 4.0 International License, which permits use, sharing, adaptation, distribution and reproduction in any medium or format, as long as you give appropriate credit to the original author(s) and the source, provide a link to the Creative Commons licence, and indicate if changes were made. The images or other third party material in this article are included in the article's Creative Commons licence, unless indicated otherwise in a credit line to the material. If material is not included in the article's Creative Commons licence and your intended use is not permitted by statutory regulation or exceeds the permitted use, you will need to obtain permission directly from the copyright holder. To view a copy of this licence, visit http://creativecommons.org/licenses/by/4.0/.

\section{References}

1. Hurley M, Vyas H (2015) Respiratory problems in children with neurodisability. Paediatr Child Health 25(10):463-466

2. Blackmore AM, Bear N, Blair E, Langdon K, Moshovis L, Steer K, Wilson AC (2018) Predicting respiratory hospital admissions in young people with cerebral palsy. Arch Dis Child 103(12):1119 1124. https://doi.org/10.1136/archdischild-2017-314346 Epub 2018 Mar 19

3. Proesmans M (2016) Respiratory illness in children with disability: a serious problem? Breathe 12:e97-e103
4. Young NL, McCormick AM, Gilbert T et al (2011) Reasons for hospital admissions among youth and young adults with cerebral palsy. Arch Phys Med Rehabil 92(1):46-50

5. Wright RE, Wright FR, Carson CA (1996) Videofluoroscopic assessment in children with severe cerebral palsy presenting with dysphagia. Pediatr Radiol 26(10):720-722

6. Owayed AF, Campbell DM, Wang EE (2000) Underlying causes of recurrent pneumonia in children. Arch Pediatr Adolesc Med 154(2):190-194

7. Weir KA, McMahon S, Taylor S (2011) Oropharyngeal aspiration and silent aspiration in children. Chest 140(3):589-597

8. Erasmus CE, van Hulst K, Rotteveel JJ, Willemsen MAAP, Jongerius PH (2012) Clinical practice: swallowing problems in cerebral palsy. Eur J Pediatr 171(3):409-414

9. Young NL, Gilbert TK, McCormick A, Ayling-Campos A, Boydell K, Law M, Fehlings DL, Mukherjee S, Wedge JH, Williams JI (2007) Youth and young adults with cerebral palsy use of physician and hospital services. Arch Phys Med Rehabil 88(6):696-702

10. Thorburn K, Jardine M, Taylor N, Reilly N, Sarginson RE, van Saene HK (2009) Antibiotic-resistant bacteria and infection in children with cerebral palsy requiring mechanical ventilation. Pediatr Crit Care Med 10(2):222-226. https://doi.org/10.1097/PCC. 0b013e31819368ac

11. Zar H, Saiman L, Quittell L, Prince A (1995) Binding of Pseudomonas aeruginosa to respiratory epithelial cells from patients with various mutations in the cystic fibrosis transmembrane regulator. J Pediatr 126(2):230-233

12. Emerson J, Rosenfeld M, McNamara S, Ramsey B, Gibson RL (2002) Pseudomonas aeruginosa and other predictors of mortality and morbidity in young children with cystic fibrosis. Pediatr Pulmonol 34(2):91-100

13. Nixon GM, Armstrong DS, Carzino R, Carlin JB, Olinsky A, Robertson CF, Grimwood K (2001) Clinical outcome after early Pseudomonas aeruginosa infection in cystic fibrosis. J Pediatr 138(5):699-704

14. Ramsey BW, Wentz KR, Smith AL, Richardson M, WilliamsWarren J, Hedges DL, Gibson R, Redding GJ, Lent K, Harris K (1991) Predictive value of oral cultures for identifying lower airways bacteria in cystic fibrosis patients. Am Rev Resir Dis 144(2): 331-337

15. Gerding CA, Tsang A, Yasseen AS, Armstrong K, McMillan HJ, Kovesi T (2016) Association between chronic aspiration and chronic airway infection with pseudomonas aeruginosa and other Gramnegative bacteria in children with cerebral palsy. Lung 194:307314

16. Yusuf et al. Emergence of antimicrobial resistance to Pseudomonas aeruginosa in the intensive care unit: association with the duration of antibiotic exposure and mode of administration Ann. Intensive Care (2017) 7:72

17. Bassetti M, Vena A, Croxatto A, Righi E, Guery B. How to manage Pseudomonas Aeruginosa infections Drugs in Context 2018; 7 : 212527. DOI: https://doi.org/10.7573/dic.212527

18. Sergio Ramírez-Estrada et al. Pseudomonas aeruginosa ventilatorassociated pneumonia management. Infect Drug Resist. 2016; 9: 7 18. Published online 2016 Jan 20

19. McCrea N, O’Donnell R, Brown R (2013) Outpatient respiratory management of the child with severe neurological impairment. Arch Dis Child Educ Pract Ed 98(3):84-91. https://doi.org/10. 1136/archdischild-2012-302324

20. Zhu H., Robertson M., Heraghty J., Bossley C. National survey on management of pseudomonas aeruginosa infection in children with neurodisability or long-term respiratory support. Embase Pediatric Pulmonology. Conference: 16th Congress of the International Pediatric Pulmonology, 2017. Portugal. 52 (Supplement 46) (pp S111) 
21. Sanders CD, Guimbellot JS, Muhlebach MS et al (2018) Tracheostomy in children: epidemiology and clinical outcomes. Pediatr Pulmonol 53(9):1269-1275. https://doi.org/10.1002/ppul. 24071

22. Lepainteur M, Ogna A, Clair B, Dinh A, Tarragon C, Prigent H, Davido B, Barbot F, Vaugier I, Afif M, Roux AL, Rottman M, Orlikowski D, Herrmann JL, Annane D, Lawrence C (2019) Risk factors for respiratory tract bacterial colonization in adults with neuromuscular or neurological disorders and chronic tracheostomy. Respir Med 152:32-36. https://doi.org/10.1016/j.rmed.2019.04.015

23. Brook I (1979) Bacterial colonization, tracheobronchitis, and pneumonia following tracheostomy and long-term intubation in pediatric patients. Chest 76(4):420-424. 2

24. Niederman MS, Ferranti RD, Zeigler A, Merrill WW, Reynolds HY (1984) Respiratory infection complicating long-term tracheostomy. The implication of persistent Gram-negative tracheobronchial colonization. Chest 85(1):39-44
25. Harlid R, Andersson G, Frostell CG, Jörbeck HJ, Ortqvist AB (1996) Respiratory tract colonization and infection in patients with chronic tracheostomy. A one-year study in patients living at home. Am J Respir Crit Care Med 154(1):124-129

26. Russell CJ, Simon TD, Mamey MR, Newth CJL, Neely MN (2017) Pseudomonas aeruginosa and post-tracheotomy bacterial respiratory tract infection readmissions. Pediatr Pulmonol 52:1212-1218

27. McCaleb R, Warren RH, Willis D, Maples HD, Bai S, O'Brien CE (2016) Description of respiratory microbiology of children with long-term tracheostomies. Respir Care 61:447-452

28. Cline JM, Woods CR, Ervin SE, Rubin BK, Kirse DJ (2012) Surveillance tracheal aspirate cultures do not reliably predict bacteria cultured at the time of an acute respiratory infection in children with tracheostomy tubes. Chest. 141(3):625-631. https://doi.org/ $10.1378 /$ chest.10-2539

Publisher's note Springer Nature remains neutral with regard to jurisdictional claims in published maps and institutional affiliations. 